\section{Tilt illusions with checkerboard and grating surrounds}

\author{
ZHANG WU-TIAN \\ Chinese Academy of Sciences \\ Peking, People's Republic of China
}

Perceptual effects obtained when checkerboards are used as stimuli have been taken as evidence that patterns are analyzed in the human visual system on the basis of spatial frequency information rather than edge characteristics. The fundamental frequency of a grating is the same orientation as the edges that make up the grating. In contrast, a checkerboard constituted of checks with vertical and horizontal edges lacks fundamental Fourier components at vertical and horizontal orientations (see Kelly, 1976). Instead, the fundamental frequency components are at an orientation of $45 \mathrm{deg}$ from that of local edges in the display. A grating of fixed luminance contrast is less visible at an oblique orientation than it is at vertical or horizontal orientations (see Appelle, 1972). However, the visibility of a checkerboard is most impaired when the edges of checks are vertical and horizontal and, hence, when the fundamental Fourier components are at an oblique orientation (Kelly, 1976). Similarly, one grating maximally masks another grating when the two gratings are identical in orientation. A checkerboard maximally masks a grating when the fundamental Fourier components of the two displays are at the same orientation, for example, when the grating is vertical and the local edges of the checkerboard are oblique (Green, 1980).

In the conventional McCollough effect, alternate exposure to 45-deg tilted lines in red light and vertical lines in green light cause 45-deg lines subsequently viewed in white light to appear green and vertical lines to look pink. However, adaptation to a checkerboard with vertical-horizontal edges in red light and a checkerboard with oblique edges in green light results in a 45-deg test grating appearing pink and a vertical grating looking green (Green, Corwin, \& Zemon, 1976; May \& Matteson, 1976). May, Agamy, and Matteson (1978) subsequently demonstrated that the McCollough effect induced with checkerboards is selective to Fourier components only when the spatial frequency is 3 cycles per degree $(\mathrm{c} / \mathrm{deg})$ or greater. It was not possible to induce McCollough effects using displays that were slightly lower in spatial frequency, and, at $.8 \mathrm{c} / \mathrm{deg}$, the aftereffect was con-

This research was completed at La Trobe University, Australia, while the author held an appointment as Visiting Research Fellow. He would like to thank Ray Over for assistance in the research and in the preparation of the manuscript. Requests for reprints should be sent to Zhang Wu-tian, Institute of Psychology, Chinese Academy of Sciences, Peking, People's Republic of China. tingent on local edge information rather than on the Fourier components of the checkerboard.

The objective of the present study was to measure and compare tilt illusions produced by checkerboards and gratings. In the classical tilt illusion, the perceived tilt of a test grating is systematically influenced by the orientation of a surround grating. The illusion is of greatest magnitude when the center and the surround differ in orientation by $10.15 \mathrm{deg}$, and the distortion is markedly less when the difference is 45 or $60 \mathrm{deg}$ (see Over, Broerse, \& Crassini, 1972). The question of interest when the test figure is a grating and the surround a checkerboard is whether the magnitude of the illusion depends on the difference in orientation between the center-surround edges or the Fourier components. In view of the demonstration by May et al. (1978) that the orientation selectivity of the McCollough effect induced with checkerboards depends on absolute spatial frequency, the present study used displays within the low and the medium spatial frequency ranges.

The observers used rotary controls to adjust the orientation of a test grating shown on a television screen. Measures were obtained when the cardboard field surrounding this grating was blank, a grating, and a checkerboard. The test grating subtended $2 \mathrm{deg} 25 \mathrm{~min}$ in diameter at a viewing distance of $105 \mathrm{~cm}$, while the surround was an annulus with an outer diameter of $6 \mathrm{deg} 30 \mathrm{~min}$. The tilt illusion was measured using squarewave gratings of contrast of .7 at four spatial frequencies: $.8,2.0,3.5$, and $5.2 \mathrm{c} / \mathrm{deg}$. The spatial frequency of a checkerboard fundamental is $\sqrt{2}$ that of a grating with bars as wide as the edge of the checkerboard. In the present study, both the surround grating and the fundamental component of the checkerboard were always maintained at the same spatial frequency as the test grating that was in use.

The observer's task on each trial was to set the test grating until it appeared vertical. The size of the illusion was measured with the surround grating and the check edges tilted $15,30,45$, and 60 deg clockwise and counterclockwise from vertical. Under each surround condition, the four observers made 16 judgments at each of the 16 combinations of orientation and spatial frequency values. In addition, eight control judgments were obtained at each spatial frequency using the blank surround. The starting position of the test grating was $10 \mathrm{deg}$ clockwise from vertical for half the settings and $10 \mathrm{deg}$ counterclockwise for the remainder.

The tilt illusion was expressed as the difference between an observer's settings to vertical when the surround was patterned and blank. These values were scored as positive when the displacement of the test grating relative to pretest settings was in the same direction as the surround edge, and as negative when the displacement was in the opposite direction. Table 1 
Table 1

Mean Size (in Degrees) of Tilt Aftereffect Induced by Grating and Checkerboard Surrounds at Different Orientations (Edges Relative to Vertical)

\begin{tabular}{ccccc}
\hline & \multicolumn{4}{c}{ Spatial Frequency (in Degrees) } \\
\cline { 2 - 5 } $\begin{array}{c}\text { Orientation } \\
\text { (in Degrees) }\end{array}$ & 0.89 & 2.00 & 3.50 & 5.00 \\
\hline \multicolumn{5}{c}{ Grating } \\
15 & 2.82 & 2.86 & 2.78 & 2.71 \\
30 & 2.17 & 2.84 & 2.39 & 2.47 \\
45 & 1.90 & 2.18 & 2.19 & 2.20 \\
60 & 1.90 & 1.65 & 1.91 & 2.29 \\
& & Checkerboard & \\
15 & 2.40 & 2.24 & 2.33 & 2.57 \\
30 & 2.36 & 2.14 & 2.07 & 1.96 \\
45 & 2.05 & 2.06 & 2.10 & 2.06 \\
60 & 1.57 & 1.75 & 1.71 & 2.47 \\
\hline
\end{tabular}

shows the mean magnitude of illusion obtained using grating and checkerboard surrounds at different spatial frequencies. All values were positive.

A checkerboard with one set of check edges tilted $15 \mathrm{deg}$ clockwise from vertical is equivalent, in Fourier terms, to gratings tilted $30 \mathrm{deg}$ counterclockwise and $60 \mathrm{deg}$ clockwise from vertical. Similarly, a display with check edges tilted $60 \mathrm{deg}$ clockwise is equivalent to gratings tilted $15 \mathrm{deg}$ clockwise and $75 \mathrm{deg}$ counterclockwise from vertical. It is clear, however, from inspection of Table 1 that the direction and magnitude of the tilt illusion varied with the difference in orientation between center and surround edges rather than Fourier components. Analysis of variance showed that, at each spatial frequency, the mean illusion varied significantly in magnitude $(\mathrm{p}<.01)$ with orientation of the surround $[\mathrm{F}(3,9)=15.80$ at $.8 \mathrm{c} / \mathrm{deg}, 9.38$ at $2.0 \mathrm{c} / \mathrm{deg}, 7.51$ at $3.5 \mathrm{c} / \mathrm{deg}$, and 9.08 at $5.0 \mathrm{c} / \mathrm{deg}$. At three spatial frequencies, the mean illusion was similar in magnitude for grating and checkerboard surrounds $[F(1,9)=2.65$ at $.8 \mathrm{c} / \mathrm{deg}, 3.20$ at $3.5 \mathrm{c} / \mathrm{deg}, .28$ at $5.0 \mathrm{c} / \mathrm{deg}$; each p $>.05$ ], and at these spatial frequencies the interaction between surround stimulus and orientation was not significant $[\mathrm{F}(3,9)=3.35$ at $.8 \mathrm{c} / \mathrm{deg}, .62$ at $3.5 \mathrm{c} / \mathrm{deg}$, and .36 at $5.0 \mathrm{c} / \mathrm{deg}$; each $\mathrm{p}>.05]$. At $2.0 \mathrm{c} / \mathrm{deg}$, the illusion was larger with the grating than with the checkerboard surround $[F(1,9)=4.05, p<.05]$, and the interaction between type of surround and orientation was significant $[F(1,9)=4.24, p<.05]$. Both of these differences reflected the unexpectedly large illusion obtained when the surround grating was tilted $30 \mathrm{deg}$ from vertical.

The tilt illusion obtained with gratings has been attributed to inhibitory interaction among the spatially selective detectors that signal center and surround properties. Since the tilt illusion is smaller when center and surround gratings differ in spatial frequency (Georgeson, 1973), it has been supposed that the interactions producing perceptual error involve detectors tuned to spatial frequency as well as orientation. On these grounds, the illusion obtained with a checkerboard surround might be expected to vary in magnitude in accord with the orientation of fundamental frequency components rather than local edge information. It was, instead, found that the latter variable was influential.

May et al. (1978) showed that whether the McCollough effect induced with checkerboards is selective to fundamental frequency components or local edge information depends on the spatial frequency of the displays that are used. In the present experiment, the orientation selectivity of the illusion with grating and checkerboard surrounds was the same at three spatial frequencies, and the interaction effect obtained at the fourth value arose from an anomalous value with the grating surround rather than selectivity in accord with Fourier expectations for the checkerboard surround. It is not clear why separate rules should apply for the McCollough effect and the tilt illusion, although there have been other reports of discrepancies between paradigms. For example, exposure to one grating impairs detection of another grating at the same orientation, but comparable masking does not occur when the target and inducing gratings are simultaneously presented in center and surround format (Klein, Stromeyer, \& Ganz, 1974). Adaptation to checkerboards yields orientation-selective masking of gratings in accord with the fundamental frequency components in the display (Green, 1980), whereas the illusion obtained with simultaneous center-surround presentation reflects orientation differences between local edge information.

\section{REFERENCES}

Appelte, S. Perception and discrimination as a function of stimulus orientation: The "oblique effect" in man and animals. Psychological Bulletin, 1972, 78, 266-278.

Georgeson, M. A. Spatial frequency selectivity of a visual tilt illusion. Nature, 1973, 245, 43-45.

Green, M. Orientation-specific adaptation: Effects of checkerboards on the detectability of gratings. Perception, 1980, 9, 369-377.

Green, M., Corwin, T. R., \& Zermon, V. A. A comparison of Fourier analysis and feature analysis in pattern-specific color aftereffects. Science, 1976, 194, 147-148.

KELLY, D. H. Pattern detection and the two-dimensional Fourier transform: Flickering checkerboards and chromatic mechanisms. Vision Research, 1976, 16, 277-289.

KLein, S., Stromeyer, C. F., \& Ganz, L. The simultaneous spatial frequency shift: A dissociation between the detection and perception of gratings. Vision Research, 1974, 14, 1421-1432.

May, J. G., Agamy, G., \& Matteson, H. H. The range of spatial frequency contingent color aftereffects. Vision Research, 1978, 18, 917-921.

MaY, J. G., \& Matteson, H. H. Spatial frequency contingent color aftereffects. Science, 1976, 194, 145-147.

Over, R., Broerse, J., \& Crassini, B. Orientation illusion and masking in central and peripheral vision. Journal of Experimental Psychology, 1972, 96, 25-31.

(Manuscript received April 27, 1982; accepted for publication May 12, 1982.) 